\title{
A statistical approach to determining responses to individual peptides from pooled-peptide ELISpot data
}

\author{
Peter Ström ${ }^{\mathrm{a}}$, Nathalie Støer ${ }^{\mathrm{a}}$, Nicola Borthwick ${ }^{\mathrm{b}}$, Tao Dong ${ }^{\mathrm{c}}$, Tomáš Hanke ${ }^{\mathrm{b}, \mathrm{c}}$, Marie Reilly ${ }^{\mathrm{a}, *}$ \\ a Department of Medical Epidemiology and Biostatistics, Karolinska Institutet, Sweden \\ b The Jenner Institute, University of Oxford, UK \\ c Weatherall institute of Molecular Medicine, Oxford, UK
}

\author{
A B S T R A C T
}

\begin{abstract}
To investigate in detail the effect of infection or vaccination on the human immune system, ELISpot assays are used to simultaneously test the immune response to a large number of peptides of interest. Scientists commonly use "peptide pools", where, instead of an individual peptide, a test well contains a group of peptides. Since the response from a well may be due to any or many of the peptides in the pool, pooled assays usually need to be followed by confirmatory assays of a number of individual peptides. We present a statistical method that enables estimation of individual peptide responses from pool responses using the Expectation Maximization (EM) algo-rithm for "incomplete data". We demonstrate the accuracy and precision of these estimates in simulation studies of ELISpot plates with 90 pools of 6 or 7 peptides arranged in three dimensions and three Mock wells for the es-timation of background. In analysis of real pooled data from 6 subjects in a HIV-1 vaccine trial, where 199 pep-tides were arranged in 80 pools if size 9 or 10, our estimates were in very good agreement with the results from individual-peptide confirmatory assays. Compared to the classical approach, we could identify almost all the same peptides with high or moderate response, with less than half the number of confirmatory tests. Our method facilitates efficient use of the information available in pooled ELISpot data to avoid or reduce the need for confirmatory testing. We provide an easy-to-use free online application for implementing the method, where on uploading two spreadsheets with the pool design and pool responses, the user obtains the estimates of the individual peptide responses.
\end{abstract}

\section{Introduction}

The ELISpot assay is a commonly used tool for studying immune responses to specific antigens by counting the number of single-cell responses in a sample of peripheral blood mononuclear cells (PBMC). The PBMC together with the studied antigen(s) are placed in wells on the assay plate and the responding cells form spots on the surface of the well. The total number of spots in a well represents the number of cells responding to the antigen (Anthony and Lehmann, 2003) and thus provides a direct estimate of the magnitude of the immune response to the antigen. Standard ELISpot plates consist of 96 wells, so that a single assay can generate data on the immune response to a large variety of antigens. When there are many potential antigens and only a small fraction of these are expected to elicit a response, investigators typically use peptide pools to improve the efficiency of the assay. Fig. 1 provides a simple illustration of a two-dimensional "matrix" pool design where 81 peptides of interest are arranged in 18 pools of 9 peptides each, with each peptide being allocated to two pools. The responses from the pools will typically not enable unique identification of peptides (as in Fig. 1(a)) and the assay proceeds with a confirmation step where some or all of the peptides in responding pools are tested individually. In the example in Fig. 1(b), peptides 31, 33, 49 and 51 would need to be tested individually so that using a pooled design reduces the testing to 22 wells $(18+4)$ instead of 81 , a significant reduction in effort and time. Higher-dimensional designs can provide better discrimination but even with a four-dimensional matrix, a confirmation step will usually be required.

Where there are no a priori peptides of interest, peptide pools are used by laboratory scientists to help separate responding peptides from nonresponding peptides without estimating the strength of the individual responses, since the observed data are the accumulated spot counts over several potentially responding peptides. In the first stage of the experiment, each well contains a pool (i.e. group) of peptides, so that if there is no response from the well, then all peptides in the pool can be eliminated from further investigation. Examination of the remaining pools rarely enables the elimination of all but a few peptides as in the simple examples above, so it is assumed that the responses observed might be due to any of the peptides and these are assayed individually at the second stage. Such an approach was used in a study of the cross-reaction of human influenza and avian flu (Lee et al., 2008). 


\begin{tabular}{|c|c|c|c|c|c|c|c|c|c|}
\hline & $\begin{array}{r}10 \\
\downarrow\end{array}$ & $\begin{array}{c}11 \\
\downarrow\end{array}$ & $\begin{array}{c}12 \\
\downarrow\end{array}$ & $\begin{array}{c}13 \\
\downarrow\end{array}$ & $\begin{array}{c}14 \\
\downarrow\end{array}$ & $\begin{array}{c}15 \\
\downarrow\end{array}$ & $\begin{array}{c}16 \\
\downarrow\end{array}$ & $\begin{array}{c}17 \\
\downarrow\end{array}$ & $\begin{array}{c}18 \\
\downarrow\end{array}$ \\
\hline Pool1 $\rightarrow$ & 1 & 2 & 3 & 4 & 5 & 6 & 7 & 8 & 9 \\
\hline Pool2 $\rightarrow$ & 10 & 11 & 12 & 13 & 14 & 15 & 16 & 17 & 18 \\
\hline Pool3 $\rightarrow$ & 19 & 20 & 21 & 22 & 23 & 24 & 25 & 26 & 27 \\
\hline Pool4 $\rightarrow$ & 28 & 29 & 30 & 31 & 32 & 33 & 34 & 35 & 36 \\
\hline Pool5 $\rightarrow$ & 37 & 38 & 39 & 40 & 41 & 42 & 43 & 44 & 45 \\
\hline Pool $6 \rightarrow$ & 46 & 47 & 48 & 49 & 50 & 51 & 52 & 53 & 54 \\
\hline Pool $7 \rightarrow$ & 55 & 56 & 57 & 58 & 59 & 60 & 61 & 62 & 63 \\
\hline Pools $\rightarrow$ & 64 & 65 & 66 & 67 & 68 & 69 & 70 & 71 & 72 \\
\hline Pool9 $\rightarrow$ & 73 & 74 & 75 & 76 & 77 & 78 & 79 & 80 & 81 \\
\hline
\end{tabular}

(a)

\begin{tabular}{|c|c|c|c|c|c|c|c|c|c|}
\hline & $\begin{array}{r}10 \\
\downarrow\end{array}$ & $\begin{array}{c}11 \\
\downarrow\end{array}$ & $\begin{array}{c}12 \\
\downarrow\end{array}$ & $\begin{array}{c}13 \\
\downarrow\end{array}$ & $\begin{array}{c}14 \\
\downarrow\end{array}$ & $\begin{array}{c}15 \\
\downarrow\end{array}$ & $\begin{array}{c}16 \\
\downarrow\end{array}$ & $\begin{array}{c}17 \\
\downarrow\end{array}$ & $\begin{array}{c}18 \\
\downarrow\end{array}$ \\
\hline Pool $1 \rightarrow$ & 1 & 2 & 3 & 4 & 5 & 6 & 7 & 8 & 9 \\
\hline Pool $2 \rightarrow$ & 10 & 11 & 12 & 13 & 14 & 15 & 16 & 17 & 18 \\
\hline Pool3 $\rightarrow$ & 19 & 20 & 21 & 22 & 23 & 24 & 25 & 26 & 27 \\
\hline Pool4 $\rightarrow$ & 28 & 29 & 30 & 31 & 32 & 33 & 34 & 35 & 36 \\
\hline Pool5 $\rightarrow$ & 37 & 38 & 39 & 40 & 41 & 42 & 43 & 44 & 45 \\
\hline Pool $6 \rightarrow$ & 46 & 47 & 48 & 49 & 50 & 51 & 52 & 53 & 54 \\
\hline Pool $7 \rightarrow$ & 55 & 56 & 57 & 58 & 59 & 60 & 61 & 62 & 63 \\
\hline Pool $8 \rightarrow$ & 64 & 65 & 66 & 67 & 68 & 69 & 70 & 71 & 72 \\
\hline Pool9 $\rightarrow$ & 73 & 74 & 75 & 76 & 77 & 78 & 79 & 80 & 81 \\
\hline
\end{tabular}

(b)

Fig. 1. Example of matrix pools generated from 81 peptides of interest. The peptides (numbered 1 to 81 in the figure) are grouped into 18 pools, represented by the 9 rows and 9 columns of the two-dimensional matrix. In (a), responses are obtained only from pool 4 and pool 13 and can thus be uniquely attributed to peptide 31 . In (b), the responses from pools $4,6,13$ and 15 do not enable unique identification of the peptide(s) eliciting the response, which could be one or more of peptides $31,33,49,51$.

Clearly the efficiency of this method relies on the pool sizes and the combinations of peptides in the various pools (Roederer and Koup, 2003). The focus of our work is to use the pooled assay responses to estimate the strength of the response to each individual peptide. These estimates can be used to characterise the immune response or to help inform more targeted testing of individual peptides at the second stage. This can result in significant gains in efficiency, especially in applications where only a small proportion of peptides stimulate a response.

In this paper, we show that the use of peptide pool responses is not limited to identifying a subset of peptides for confirmatory testing, but these pool responses can be used to achieve estimates of the strength of a subject's response to the unique individual peptides in the pools. Since the data available are the combined responses to the pool of peptides in each well, but interest is focused on the response to an individual peptide, we can express this as an incomplete data problem (Dempster et al., 1977). The estimates of interest can then be obtained using the Expectation Maximization (EM) algorithm, which under some reasonable assumptions reduces to the solution of simple matrix equations. In Section 2, we describe the data that motivated this work, present our statistical method in detail and describe the simulation set-up we used to investigate its performance. In Section 3 we present the results of the simulation study and illustrate the method with an application to IFN- $\gamma$ ELISpot assays of the immune responses of 6 participants in a HIV-1 vaccine trial for whom 199 peptides were assayed in 80 pools of size 9 or 10 . We also compare our results to the individual-peptide confirmatory assays conducted for the same subjects. In Section 4 we discuss the method and outline the potential for further work in this area.

\section{Materials and methods}

This work was motivated by data from IFN- $\gamma$ ELISpot assays con-

ducted during a double-blind, randomised block, phase I HIV-1 vaccine trial (Borthwick et al., 2014) where three candidate vaccines (pSG2.HIVconsv DNA, ChAdV63. HIVconsv and MVA.HIVconsv) were administered to healthy, uninfected, low-risk adults. After a small safety arm, three groups of 10 volunteers were randomised to heterologous vaccine regimens or placebo in a ratio 8:2. For the assessment of vaccine immunogenicity, investigators arranged 199 15-mer peptides overlapping by 11 amino acids in 80 pools of size 9 or 10, in a four-dimensional matrix design. The remaining wells of the plate were used for positive and negative controls. In this paper, we analyse six vaccinated subjects in one of the randomisation arms, for whom individual assays (with one peptide per well) were conducted on specimens taken on the same day post-vaccination.

Vaccine induced responses were measured using an IFN- $\gamma$ ELISpot assay on cryopreserved PBMC taken one week after the final MVA.HIVconsv immunisation (Borthwick et al., 2014). Briefly, cells were thawed and Benzonase Nuclease (Novagen) added prior to washing to degrade liberated DNA and RNA and prevent cell clumping. The number of viable cells was determined by exclusion of Trypan Blue (Sigma-Aldrich Ltd). For all samples the cell viability on the day of the thaw was $>90 \%$. For the matrix assay, cells were cultured at $1.0 \times 10^{5}$ cells per well of a 96 well ELISpot plate (S5EJ044, Merk-Millipore) coated with anti-human IFN- $\gamma$ antibody (Mabtech) and peptide pools used at $1.5 \mu \mathrm{g} / \mathrm{ml}$ per peptide. For assays using individual peptides, the cells were cultured at $0.5-1.0 \times 10^{5}$ per well depending on cell availability and each individual peptide used at $1.5 \mu \mathrm{g} / \mathrm{ml}$. Controls were (a) negative; culture medium containing 1\% DMSO but no peptide, (b) positive; phytohaemaglutinin (PHA: Sigma-Aldrich) at $10 \mu \mathrm{g} / \mathrm{ml}$ (c) HIVconsv peptide pools 1-6 (d) external positive; control cell line, NKL, that releases IFN- $\gamma$ in response to stimulation with PMA/Ionomycin (SigmaAldrich). For both assays the cells were cultured overnight and the number of IFN- $\gamma$ secreting cells identified using biotin-conjugated secondary antibodies in association with streptavidin-ALP and BCIP/NBT Plus substrate (all from Mabtech). Spot forming units (SFU) were counted on an AID ELISpot Reader (AID GmbH) using version 5 software. All results were adjusted to SFU per million PBMC after subtraction of the background negative control.

We use our method to estimate the responses of the six subjects to individual peptides from their pooled assay responses and validate our estimates against the individual confirmatory assays.

\section{Theory and calculation}

\subsection{Statistical methods}

The measure of response in an ELISpot assay, spot-forming-units (SFU), represents the number of cells that release the cytokine in response to the stimulating peptide(s). We model this underlying response of an individual to a specific peptide $i,(i=1 \ldots n)$, as a Poisson random variable, with intensity parameter $\beta_{i}$ which we refer to as the "peptide effect". Let $b_{i j}$ denote the (unobserved) response to peptide $i$ in pool $j$. Then assuming independence of the individual responses within the same pool, the likelihood of the complete unobserved data $b_{i j}$ is

$L(\beta)=\prod_{i=1}^{n} \prod_{\left\{j \mid i \in P_{j}\right\}} e^{-\beta_{i}} \frac{\beta_{i}^{b_{i j}}}{b_{i j} !}$

where $P_{j}$ denotes the set of peptides in pool $j$. 
The pool design can be represented by a matrix, $\mathrm{X}$, with element $x_{j i}=1$ if peptide $i$ belongs to pool $j$, and $x_{j i}=0$ otherwise. For example, for 200 peptides arranged in 93 pools, the design matrix $\mathrm{X}$ is given by

$\mathrm{X}=\left[\begin{array}{lllll}x_{1,1} & x_{1,2} & \cdots & \cdots & x_{1,200} \\ x_{2,1} & x_{2,2} & \cdots & \cdots & x_{2,200} \\ \vdots & \vdots & \ddots & & \vdots \\ \vdots & \vdots & & \ddots & \vdots \\ x_{93,1} & x_{93,2} & \cdots & \cdots & x_{93,200}\end{array}\right]$

where the 1 's in each row identify the peptides in that pool. We can express the maximum likelihood estimate (MLE) of a peptide effect as the average of the responses from the replicates:

$\beta_{i}=\frac{\sum_{\left\{j \mid i \in P_{j}\right\}} b_{i j}}{\sum_{\left\{j \mid i \in P_{j}\right\}} x_{i j}}$.

However, the individual responses, $b_{i j}$, are unobserved and the available data are the pool responses, $y_{j}$, which we assume to be the sums of the peptide responses within each pool

$y_{j}=\sum_{\left\{i \mid i \in P_{j}\right\}} b_{i j}$

The Expectation-Maximization (EM) algorithm (Dempster et al., 1977) provides a method of finding maximum likelihood estimates from such incomplete data by maximizing the expected log-likelihood of the complete (unobserved) data conditional on the observed data. For a given expected value of the unobserved data, the M-step maximises the conditional likelihood to obtain an estimate of the target parameter $\beta$, and for a given estimate of $\beta$ the E-step calculates the expected values of the responses. Thus the algorithm proceeds iteratively to convergence.

For the scenario considered here, the M-step of the EM algorithm is simply Eq. (2) evaluated at the latest estimate of the individual responses $\beta$, with the unobserved $b_{i j}$ replaced by their expected values conditional on the pool responses (i.e. the observed data). In the Estep, conditioning on the pool responses, which are the sums of independent Poisson variables, results in a multinomial distribution for the individual unobserved $b_{i j}$ whose conditional expected value can thus be written as.

$E\left[B_{i j} \mid y_{j}, \beta\right]=p_{i} y_{j}$

where

$p_{i}=\frac{\beta_{i}}{\sum_{i} x_{j i} \beta_{i}}$.

\subsection{Iterative solution}

Given a starting value $\beta^{(0)}$ for the vector of individual peptide responses, we can combine the M-step and E-step from Eqs. (2) and (3) into a single expression

$\beta_{i}^{(1)}=\frac{\sum_{\left\{j \mid i \in P_{j}\right\}} E\left[B_{i j} \mid y_{j}, \beta^{(0)}\right]}{\sum_{\left\{j \mid i \in P_{j}\right\}} x_{i j}}=\frac{\sum_{\left\{j \mid i \in P_{j}\right\}} \frac{\beta_{i}^{(0)}}{\sum_{i} x_{j i} \beta_{i}^{(0)}} y_{j}}{\sum_{\left\{j \mid i \in P_{j}\right\}} x_{i j}}$, to compute the updated estimate $\beta_{i}^{(1)}$ which is then updated in successive iterations until convergence to the MLE has been achieved. In matrix notation, the updating Formula (4) can be expressed as

$\beta^{(1)}=\mathrm{X}^{T}\left[y / \mathrm{X} \beta^{(0)}\right] *\left[\beta^{(0)} / \mathrm{X}^{T} 1\right]$

where "/" and "*" denote element-wise division and multiplication respectively and 1 denotes a vector with all elements equal to 1 and whose length is equal to the number of pools.

\subsection{Accommodating background noise}

Even without stimulating a well, some spots usually form which we refer to as the background. Some wells of the plate (typically at least three) are reserved as negative controls and their average used to estimate this background. Immune responses for an antigen are normally reported as "net responses", obtained by subtracting this background estimate from the average of replicate wells containing the antigen. In our modeling approach, we also wish to estimate the "net" spot count attributable to the peptide(s) and we do this explicitly by modeling the background as an independent Poisson process. This background process generates the spot counts in the negative control wells and contributes to the total spot count in all stimulated wells. We accommodate the background by an additional rate parameter $\beta_{0}$ in our model and by extending the design matrix, $\mathrm{X}$, to include an extra column with all elements equal to 1 ( since the "non-peptide" background is in every well) and an extra row for each negative control well with all elements 0 except for column 1 (since these wells only contain the non-peptide background). The parameter vector $\beta$ is thus of length $n+1$ where $n$ is the number of peptides and the first element $\beta_{0}$ can be thought of as an intercept term. For 96-well plates with three negative control wells and 200 peptides arranged in 93 pools, the extended design matrix is as follows.

$\nearrow_{\text {extended }}=\left[\begin{array}{c|ccccc}1 & x_{1,1} & x_{1,2} & \cdots & \cdots & x_{1,200} \\ 1 & x_{2,1} & x_{2,2} & \cdots & \cdots & x_{2,200} \\ \vdots & \vdots & \vdots & \ddots & & \vdots \\ \vdots & \vdots & \vdots & & \ddots & \vdots \\ 1 & x_{93,1} & x_{93,2} & \cdots & \cdots & x_{93,200} \\ \hline 1 & 0 & 0 & \cdots & \cdots & 0 \\ 1 & 0 & 0 & \cdots & \cdots & 0 \\ 1 & 0 & 0 & \cdots & \cdots & 0\end{array}\right]$

\subsection{Simulation of pooled ELISpot data}

To illustrate the method we simulated data similar to those collected in real applications (Borthwick et al., 2014; Addo et al., 2003). The parameters representing the intensity of the true response to an individual peptide were therefore either set to zero (corresponding to non-responses) or drawn from a Gamma distribution. The Gamma distribution allows us to represent a distribution of positive values with a small tail of extremes, thus providing a realistic model for assays that are directed at identifying a small number of strong responses. We used two Gamma distributions, one with shape parameter 4 and scale 10 and one with shape 6 and scale 10, representing mild and moderate responses respectively. We created a vector of parameter values, $\beta$, of length 200 to represent the true underlying immunogenicity of 200 peptides, consisting of 8 values (i.e. $4 \%$ ) drawn from one of the two Gamma distributions and 192 (96\%) zeros, permuted in random order. From these underlying rates, and assuming Poisson processes, we generated a vector $b_{1}$ of 200 observed responses. We then allocated $b_{1}$ into 30 disjoint pools of size 6 or 7 so that any specific peptide only belonged to one 
pool and the response $y_{j}$ from any pool was defined as the sum of the individual responses in the pool. Using the same underlying rates, we created two further vectors of responses, $b_{2}$ and $b_{3}$, and allocated these into disjoint pools using different combinations of peptides. The 90 pools thus belonged to three blocks, with no overlap of pools within a block. The elements of the $y$ vector of observed pool responses can be written:

$y_{j}=\mathrm{X}_{j} b_{1}, \quad j=1, \ldots, 30$

$y_{j}=\mathrm{X}_{j} b_{2}, \quad j=31, \ldots, 60$

$y_{j}=\mathrm{X}_{j} b_{3}, \quad j=61, \ldots, 90$,

where $\mathrm{X}_{j}$ indicates row $j$ of the design matrix describing the pools. The individual elements of the $b$ vectors are the "complete data" which are unknown and are to be estimated from the incomplete $y$ responses using the EM approach to obtain maximum likelihood estimates.

The final step in generating the observed pooled data was to add "noise" to each of the values $y_{j}$ and to generate 3 additional responses representing the no-peptide "negative control" or "Mock" wells. We used a Poisson variable with mean 5 to simulate noise of a level that is reasonable in HIV-1 applications that use 250,000 cells in each well. Thus our vector of observations $y$ was of length 93 , with the first 90 values representing the sum of spots from the peptides in a pool plus the additional noise, and the last 3 values representing only noise.

The observed data described above was simulated 200 times and for each realisation, the individual peptide responses were obtained by applying the EM algorithm as described. We report the average estimates over the 200 simulations together with the $95 \%$ confidence intervals estimated from the upper and lower percentiles.

Since our method assumes the data are from underlying Poisson processes, we used our simulation set-up to conduct a sensitivity analysis by investigating the performance when there is overdispersion. Similar to previous published work (Moodie et al., 2006; Dittrich and Lehmann, 2012), we generated both Poisson data $(\varphi=1)$ and negative binomial data with moderate $(\varphi=4)$ dispersion parameter.

Since a priori we assume all peptides are equally likely to elicit a response, we let the starting values for $\beta$ be $\beta^{0}=(1,1, \ldots, 1)^{T}$ and investigated the sensitivity of the EM solution by trying several different starting values for the algorithm.

\subsection{Software}

All simulations and analysis were conducted in $\mathrm{R}$ version 3.1 .1 ( $\mathrm{R}$ Development Core Team, 2007) a freely-available statistical software package. The EM method was programmed using the matrix operations described above. We used the R Shiny (RStudio, 2014) tools to program a user-friendly online application which estimates the individual peptide effects (and confidence intervals if required) when the user uploads two spreadsheets: a design file indicating which peptides belong to each pool and a response file giving the total spot count from each pool. Test data are provided on the web page, together with a thorough explanation (https://elispot.shinyapps.io/Shiny/).

\section{Results}

\subsection{Simulation results}

Table 1 displays the results of our simulations. For each of the $\beta$ values drawn from the Gamma distributions representing low and moderate intensities, we summarise the results for data simulated from a Poisson process with and without overdispersion. The average estimates of the underlying intensities from the 200 simulations are presented with the empirical $95 \%$ confidence intervals, and the average bias and percentage bias are also reported. The results indicate very good accuracy for all except the lowest intensities. The estimates with confidence intervals are presented graphically in Fig. 2.

\section{Table 1}

Summary of individual peptide responses in the simulation study, based on 200 simulations. Confidence intervals and absolute bias (with percentage bias in parentheses).

\begin{tabular}{|c|c|c|c|c|c|}
\hline & \multirow[b]{3}{*}{$\beta$} & \multicolumn{2}{|c|}{ Poission variance } & \multicolumn{2}{|c|}{ Overdispersion ${ }^{a}$} \\
\hline & & Estimate (95\% & & Estimate (95\% & \\
\hline & & $\mathrm{CI})$ & Bias & CI) & Bias \\
\hline \multirow[t]{8}{*}{ Low responses } & 73 & $71(61,82)$ & $-2(-3 \%)$ & $70(53,87)$ & $-3(-4 \%)$ \\
\hline & 67 & $65(54,75)$ & $-2(-3 \%)$ & $65(47,84)$ & $-2(-3 \%)$ \\
\hline & 60 & $58(48,68)$ & $-2(-3 \%)$ & $57(39,75)$ & $-3(-4 \%)$ \\
\hline & 38 & $37(29,46)$ & $-1(-2 \%)$ & $37(21,55)$ & $-1(-3 \%)$ \\
\hline & 33 & $31(24,38)$ & $-2(-6 \%)$ & $30(19,43)$ & $-3(-10 \%)$ \\
\hline & 29 & $29(20,40)$ & $0(1 \%)$ & $27(12,48)$ & $-2(-6 \%)$ \\
\hline & 25 & $22(15,30)$ & $-3(-10 \%)$ & $21(9,34)$ & $-4(-18 \%)$ \\
\hline & 19 & $16(8,24)$ & $-3(-13 \%)$ & $15(3,29)$ & $-4(-20 \%)$ \\
\hline \multirow{8}{*}{$\begin{array}{l}\text { Medium } \\
\text { responses }\end{array}$} & 108 & $106(92,117)$ & $-2(-2 \%)$ & $104(81,129)$ & $-4(-4 \%)$ \\
\hline & 86 & $84(74,95)$ & $-2(-3 \%)$ & $82(62,107)$ & $-4(-5 \%)$ \\
\hline & 73 & $70(60,81)$ & $-3(-4 \%)$ & $69(48,88)$ & $-4(-5 \%)$ \\
\hline & 58 & $55(45,65)$ & $-3(-5 \%)$ & $53(38,69)$ & $-5(-9 \%)$ \\
\hline & 52 & $51(41,63)$ & $-1(-2 \%)$ & $51(35,70)$ & $-1(-1 \%)$ \\
\hline & 37 & $35(26,44)$ & $-2(-4 \%)$ & $33(17,50)$ & $-4(-11 \%)$ \\
\hline & 34 & $32(25,40)$ & $-2(-4 \%)$ & $31(18,44)$ & $-3(-9 \%)$ \\
\hline & 28 & $24(16,32)$ & $-4(-13 \%)$ & $23(10,39)$ & $-5(-19 \%)$ \\
\hline
\end{tabular}

a Overdispersion parameter set to 4 (i.e. variance 4 times as high as for Poisson).

\subsection{Application to analysis of peptide pool ELISpot assays}

We illustrate our method with an application to data from a HIV-1 vaccine trial which investigated the responses of participants to 80 pools of 9 or 10 peptides per pool, arranged in four blocks (Borthwick et al., 2014). The maximum likelihood estimates of the responses to the 199 individual peptides were obtained by applying the matrix Eq. (5). We tried several starting values for initiating the EM algorithm and all of these converged to practically the same estimates (absolute difference of less than one spot).

Using the sum of the estimated individual peptide responses to predict the pool responses, we found good agreement between predicted and observed pool responses with increasing variance for increasing spot counts as expected for Poisson variables (Supplementary Fig. 1). To validate our findings, we compared our method to the usual twostage approach, where any peptide in a pool testing negative at the first stage is eliminated and the remaining peptides subjected to confirmatory testing at the second stage. To identify peptides that would have been selected for re-testing by either the classical approach or the EM approach we defined a positive pool or positive EM estimate using a standard definition in HIV applications, i.e. a net response of $>50 \mathrm{SFU} /$ $10^{6}$ and at least 4 times background. Table 2 compares the performance of the naive and EM methods in detecting peptides with "true" cultured assay response above various thresholds. For comparison, the table also presents the detection rates for complete testing of all 199 individual peptides. These results demonstrate that the sensitivity of both methods drops for lower levels of response. Although both methods identify most of the same peptides for moderate or high cut-offs, the EM approach manages to do this with much fewer tests.

Inspection of the magnitude of the estimated responses on QQ plots indicated that all except volunteer number 409 appeared to have one or two peptides with markedly high response (Supplementary Fig. 2(a)). In Supplementary Fig. 2(b), we present the responses on a Manhattan-type plot which indicates that some peptides stimulate a response in more than one individual while others are associated with "private" responses.

The scatter plots in Supplementary Fig. 3 illustrate good agreement between our estimates and the measured individual response: subjects 404 and 410 each had one high response which was identified, while subjects 405, 409 and 411 had several high responses of which 4, 5 and 5 , respectively, out of the 5 largest responses were identified. For subject 406 we identified two large responses which did not agree with the two highest responses in the cultured assay. 


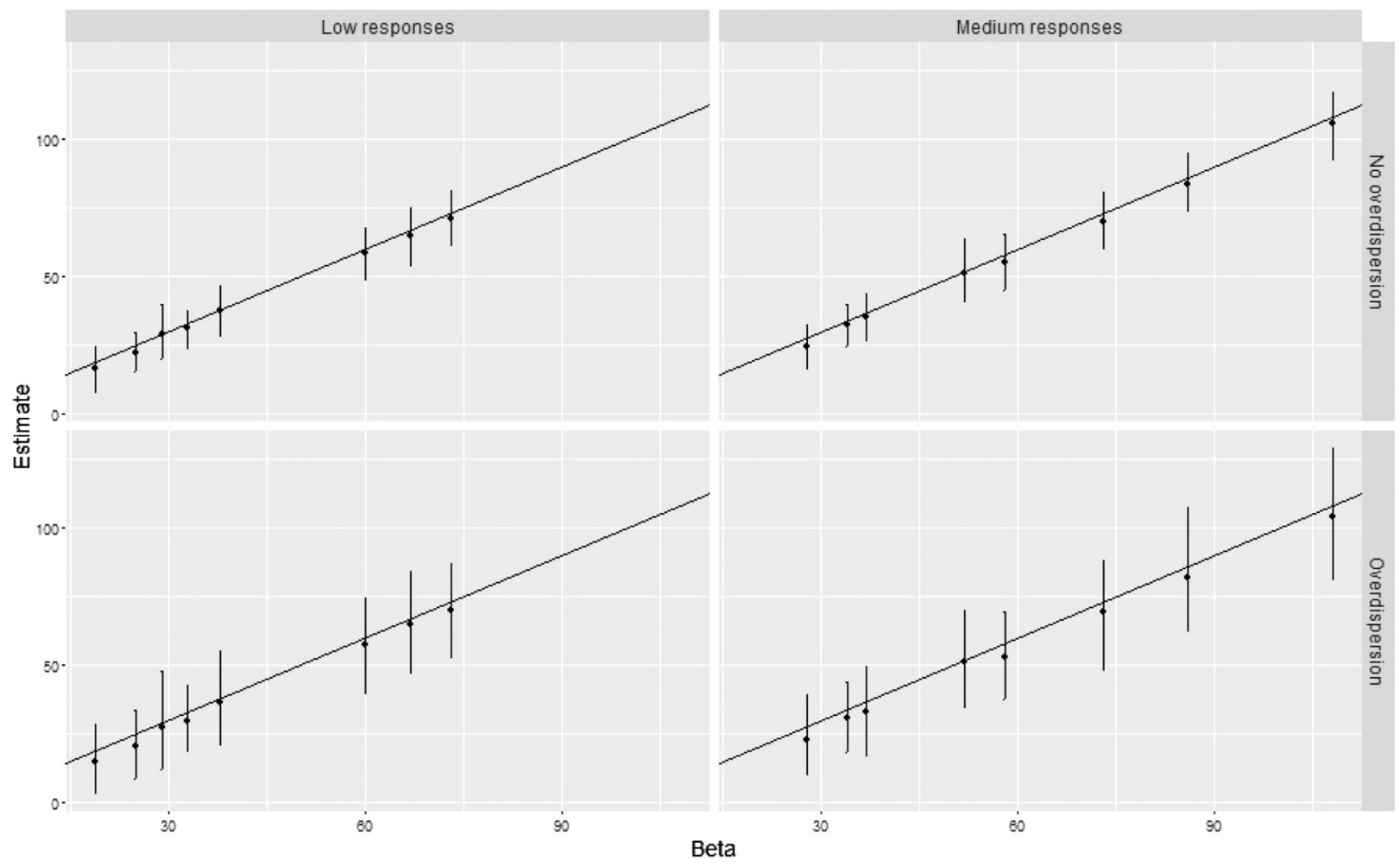

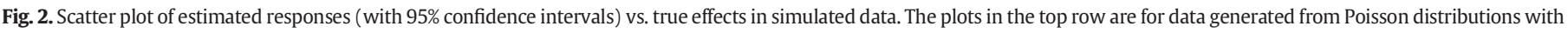
low (left) and moderate (right) levels of response. Bottom row plots are for overdispersed Poisson data.

Table 2

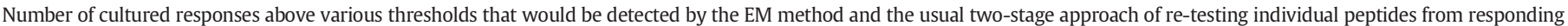
pools.

\begin{tabular}{|c|c|c|c|c|}
\hline Positivity criterion & Subject & EM & Naive & Test all \\
\hline \multirow[t]{7}{*}{$>500$} & Subject 404 & $1 / 2$ & $1 / 1$ & $1 / 199$ \\
\hline & Subject 405 & $4 / 9$ & $4 / 24$ & $5 / 199$ \\
\hline & Subject 406 & $0 / 19$ & $0 / 52$ & $1 / 199$ \\
\hline & Subject 409 & $5 / 6$ & $5 / 7$ & $5 / 199$ \\
\hline & Subject 410 & $0 / 5$ & $0 / 2$ & $0 / 199$ \\
\hline & Subject 411 & $7 / 32$ & 7/75 & 7/199 \\
\hline & Total & $17 / 73$ & $17 / 161$ & $19 / 1194$ \\
\hline \multirow[t]{7}{*}{$>400$} & Subject 404 & $1 / 2$ & $1 / 1$ & 1/199 \\
\hline & Subject 405 & $6 / 9$ & $6 / 24$ & 7/199 \\
\hline & Subject 406 & $0 / 19$ & $1 / 52$ & $2 / 199$ \\
\hline & Subject 409 & $6 / 6$ & $6 / 7$ & 9/199 \\
\hline & Subject 410 & $0 / 5$ & $0 / 2$ & 0/199 \\
\hline & Subject 411 & $9 / 32$ & $9 / 75$ & 9/199 \\
\hline & Total & $22 / 73$ & $23 / 161$ & $28 / 1194$ \\
\hline \multirow[t]{7}{*}{$>300$} & Subject 404 & $1 / 2$ & $1 / 1$ & $1 / 199$ \\
\hline & Subject 405 & $7 / 9$ & $8 / 24$ & 9/199 \\
\hline & Subject 406 & $0 / 19$ & $1 / 52$ & 5/199 \\
\hline & Subject 409 & $6 / 6$ & $6 / 7$ & $18 / 199$ \\
\hline & Subject 410 & $0 / 5$ & $0 / 2$ & 0/199 \\
\hline & Subject 411 & $11 / 32$ & $11 / 75$ & $12 / 199$ \\
\hline & Total & $25 / 73$ & $27 / 161$ & $45 / 1194$ \\
\hline \multirow[t]{7}{*}{$>200$} & Subject 404 & $1 / 2$ & $1 / 1$ & 2/199 \\
\hline & Subject 405 & $8 / 9$ & $8 / 24$ & $13 / 199$ \\
\hline & Subject 406 & $1 / 19$ & $2 / 52$ & $6 / 199$ \\
\hline & Subject 409 & $6 / 6$ & $6 / 7$ & $54 / 199$ \\
\hline & Subject 410 & $1 / 5$ & $1 / 2$ & 1/199 \\
\hline & Subject 411 & $16 / 32$ & $18 / 75$ & $22 / 199$ \\
\hline & Total & $33 / 73$ & $36 / 161$ & $98 / 1194$ \\
\hline \multirow[t]{7}{*}{$\mathrm{All}^{\mathrm{a}}$} & Subject 404 & $2 / 2$ & $1 / 1$ & $12 / 199$ \\
\hline & Subject 405 & $8 / 9$ & $10 / 24$ & 28/199 \\
\hline & Subject 406 & $4 / 19$ & $7 / 52$ & 12/199 \\
\hline & Subject 409 & $6 / 6$ & $7 / 7$ & $104 / 199$ \\
\hline & Subject 410 & $2 / 5$ & $1 / 2$ & 5/199 \\
\hline & Subject 411 & $24 / 32$ & $39 / 75$ & $61 / 199$ \\
\hline & Total & $46 / 73$ & $65 / 161$ & $222 / 1194$ \\
\hline
\end{tabular}

${ }^{\text {a }}$ Net response above $50 \mathrm{SFU} / 10^{6}$ and at least 4 times background. 


\section{Discussion}

We have shown that it is possible, in realistic situations, not only to separate potentially responding peptides from non-responding peptides using peptide pools, but to achieve reliable estimates of the strength of each response. We illustrated how the unobserved responses of individual peptides in peptide pools can be expressed as an incomplete data problem. We have derived the equations for estimating these individual effects using the EM algorithm and shown how these require just simple matrix operations for their solution. Simulation studies demonstrated that the resulting estimates had good accuracy. There was relatively small bias in the estimates, typically $<10 \%$, with larger bias occurring only for peptides with small effects.

In an application to data from a HIV-1 vaccine trial where validation data were available from individual cultured assays, we compared the performance of our approach to the classical re-testing approach. The two methods identified almost all the same peptides with moderate or high response $\left(>200 \mathrm{SFU} / 10^{6}\right.$ ) but with the EM method subjecting fewer peptides to confirmatory testing. The sensitivity of both methods reduces for lower level responses, with the EM approach being consistently more efficient, requiring less than half the number of confirmatory tests conducted by the naive method. For the identification of any response meeting the minimal criteria, the naive approach detects many more, but at a high cost in terms of numbers of tests required. Thus the choice of method needs to take into account the balance between the user's willingness to pay for many tests versus the cost of missing some true responses of lower magnitude.

Our estimates were in good agreement with the measured individual responses for 5 of the 6 subjects studied, but for one subject the two largest of the estimated and observed individual responses were discordant. It is of interest to note that neither of the two peptides with high response only in the cultured assay would have been found by the usual re-testing approach as the pools containing them had low or very low responses that would have eliminated these peptides from further testing. These peptides would also not have been found by our approach which considered the combined (low) evidence of all the pool values. Thus, these two responses only came to light because of the exhaustive testing of all individual peptides in the cultured assays. The expansion of individual $\mathrm{T}$ cell specificities for cultured assays, while increasing the sensitivity of detection, can also result in a small percent of spots from other (NK) cells that are not stimulated by that peptide (Ahmed et al., 2016). The two peptides that we estimated to have the highest responses but for which the cultured assay found no/low responses, belonged to pools with moderate responses and so would have been selected for confirmatory testing, in which case one of them would have been "found". This is consistent with our observation of low sensitivity in the confirmatory testing of peptides with true responses of this order of magnitude $\left(<200 \mathrm{SFU} / 10^{6}\right)$.

In the last decade, statistical methods have made a significant contribution to prediction in immunological research (Nielsen et al., 2004; Nielsen and Marcatili, 2015; Korber et al., 2006), including pooled-peptide mapping in HIV studies (Fiore-Gartland et al., 2016). Our method provides a statistical solution and software for estimating the individual peptide responses from peptide pool data. Using our on-line application, the user has simply to upload a design file and a results file that specify for each pool the constituent peptides and the responses respectively. The estimates of the individual peptide responses (and confidence intervals if required) will then be presented on the screen and provided in a spreadsheet for download.

We model the underlying response rates of each person to each individual peptide as Poisson random variables. If instead our focus was the response to a specific peptide on the population level, or the distribution of one person's responses to a large number of peptides, then a zero-inflated Poisson distribution may be more reasonable. While we illustrated our algorithm with an application to HIV data, it has a wide range of applications, as pooled ELISpot assays are used in many areas of medical research to help characterise the immune response and inform development of vaccines.

A limitation of our method is that we assume additivity of the individual responses in a pool. In HIV applications, efforts are made to design peptide pools in a way that even allows pool responses to be added to determine the total response (Mwau et al., 2002). Thus the assumption of additivity underlies the interpretation of pooled assay responses and consequently the detection of individual responses, and not just the method we propose for their estimation. Situations where this assumption would be violated include immunodominance (Im et al., 2011). In such cases, if the response suppressed in one pool is detectable in another, then the individual peptide response might still be identified either by classical confirmatory testing or by our algorithm. However, if the response of the pool with immunosuppression is lower than the criterion defining a minimal response, then the individual (suppressed) peptide response will not be found by the classical method since that approach involves elimination from further testing of all peptides in a non-responding pool. In contrast, with the EM method, all pool responses enter the estimation, so although the dampening of a response due to immunodominance will result in reduced sensitivity of detecting low responses, higher responses might still be detectable. Non-additivity might also need to be considered in some special applications where there is known cross-reaction between peptides (Zhao et al., 2007) or where a pool contains a peptide that is suspected of giving a false positive result (Goonetilleke et al., 2006).

In common with many other high-throughput assays, ELISpot assays may have variation due to the design of the plate. For example, there could be a "block" effect from the different groups of pools if these used different reagents or the added specimens differed in some systematic way (e.g. date of sampling, number of cells). Our method can be readily extended to accommodate such features by weighting the working vector in Eq. (5) by the appropriate block effects and modifying the matrix $X$ accordingly. Further work is needed to quantify the uncertainty in the estimates of individual peptide responses, which in addition to the variation expected from a Poisson process, may have overdispersion due to technical variation. There may also be applications where the normalisation of the ELISpot assays requires more elaborate models such as those used in other areas of immunology (Nahtman et al., 2007).

Expressing this problem in a statistical framework opens the way for more efficient use of pooled ELISpot assays in screening for biomarkers or in identifying vaccine targets in a range of applications. In contrast to the usual practice of classifying an individual's response to a specific peptide as positive or negative based on simple decision rules applied to the SFU (John-Stewart et al., 2009), the method presented here enables the estimation of the number of SFU per million PBMC generated by the individual to different peptides. Pooled assays to obtain these estimates could also be used as a first step in experiments where the focus is the prevalence of responders in the population: by using the estimates rather than individual assay results as input to available algorithms (Beissbarth et al., 2005), the population parameters of interest could be estimated at a much reduced cost. In addition to facilitating the use of the pooled ELISpot assay data currently generated, our method also provides a statistical framework for further work on the optimal design of pooled assays (i.e. the choice of pool sizes and the pattern of their overlap) to maximise efficiency.

\section{Acknowledgements}

This work was supported by the European and Developing Countries Clinical Trials Partnership (EDCTP), Grants CT.2006.33111.002 and SP.2011.41304.022, with co-funding from the Swedish International Development Cooperation Agency (SIDA). The work was also jointly funded by the UK Medical Research Council and the UK Department for International Development (DFID) under the MRC/DFID Concordat agreements (G0701669 and G1001757). 


\section{Appendix A. Supplementary figures}

Supplementary data to this article can be found online at http://dx. doi.org/10.1016/j.jim.2016.05.006.

\section{References}

Addo, M.M., Yu, X.G., Rathod, A., et al., 2003. Comprehensive epitope analysis of human immunodeficiency virus type 1 (HIV-1)-specific T-cell responses directed against the entire expressed HIV-1 genome demonstrate broadly directed responses, but no correlation to viral load. J. Virol. 77 (3), 2081-2092. http://dx.doi.org/10.1128/ JVI.77.3.2081-2092.2003.

Ahmed, T., Borthwick, N.J., Gilmour, J., Hayes, P., Dorrell, L., Hanke, T., 2016. Control of HIV-1 replication in vitro by vaccine-induced human CD8 + T cells through conserved subdominant Pol epitopes. Vaccine 34, 1215-1224. http://dx.doi.org/10. 1016/j.vaccine.2015.12.021.

Anthony, D.D., Lehmann, P.V., 2003. T-cell epitope mapping using the ELISpot approach. Methods 29 (3), 260-269. http://dx.doi.org/10.1016/S1046-2023(02)00348-1.

Beissbarth, T., Tye-Din, J.A., Smyth, G.K., et al., 2005. Modelling and analysis of T-cell epitope screening data. Proceedings of the 20th International Workshop on Statistical Modelling, pp. 101-109.

Borthwick, N., Ahmed, T., Ondondo, B., et al., 2014. Vaccine-elicited human T cells recognizing conserved protein regions inhibit HIV-1. Mol. Ther. 22 (2), 464-475. http://dx. doi.org/10.1038/mt.2013.248

Dempster, A.P., Laird, N.M., Rubin, D.B., 1977. Maximum likelihood from incomplete data via the EM algorithm. J. R. Stat. Soc. Ser. B 39 (1), 1-38. http://dx.doi.org/10.1016/ S0022-1759(02)00423-4.

Dittrich, M., Lehmann, P.V., 2012. Statistical analysis of ELISpot assays. Methods Mol. Biol. 792, 173-183.

Fiore-Gartland, A., Manso, B.A., Friedrich, D.P., et al., 2016. Pooled-peptide epitope mapping strategies are efficient and highly sensitive: an evaluation of methods for identifying human T cell epitope specificities in large-scale HIV vaccine efficacy trials. PLoS One 11 (2), e0147812. http://dx.doi.org/10.1371/journal.pone.0147812.

Goonetilleke, N., Moore, S., Dally, L., et al., 2006. Induction of multifunctional human immunodeficiency virus type 1 (HIV-1)-specific T cells capable of proliferation in healthy subjects by using a prime-boost regimen of DNA- and modified vaccinia virus Ankara-vectored vaccines expressing HIV-1 gag coupled to CD8 + T-cell epitopes. J. Virol. 80 (10), 4717-4728. http://dx.doi.org/10.1128/JVI.80.10.4717-4728. 2006.

Im, E.J., Hong, J.P., Roshorm, Y., et al., 2011. Protective efficacy of serially up-ranked subdominant CD8 + T cell epitopes against virus challenges. PLoS Pathog. 7 (5), e1002041. http://dx.doi.org/10.1371/journal.ppat.1002041.

John-Stewart, G.C., Mbori-Ngacha, D., Payne, B.L., et al., 2009. HIV-1-specific cytotoxic T lymphocytes and breast milk HIV-1 transmission. J. Infect. Dis. 199 (6), 889-898. http://dx.doi.org/10.1086/597120.

Korber, B., LaBute, M., Yusim, K., 2006. Immunoinformatics comes of age. PLoS Comput. Biol. 2 (6), e71. http://dx.doi.org/10.1371/journal.pcbi.0020071.

Lee, L.Y.-H., Ha, D.L.A., Simmons, C., et al., 2008. Memory T cells established by human influenza A infection cross-react with avian influenza A (H5N1) in healthy individuals. J. Clin. Investig. 118 (10), 3478-3490. http://dx.doi.org/10.1172/JCI32460.

Moodie, Z. Huang Y. Gu, L, et al, 2006. Statistical positivity criteria for the analysis of ELISpot assay data in HIV-1 vaccine trials. J. Immunol. Methods 315 (1-2), 121-132. http://dx.doi.org/10.1016/j.jim.2006.07.015.

Mwau, M., McMichael, A.J., Hanke, T., 2002. Design and validation of an enzyme-linked immunospot assay for use in clinical trials of candidate HIV vaccines. AIDS Res. Hum. Retrovir. 18 (9), 611-618.

Nahtman, T., Jernberg, A., Mahdavifar, S., et al., 2007. Validation of peptide epitope microarray experiments and extraction of quality data. J. Immunol. Methods 328 (1-2), 1-13. http://dx.doi.org/10.1016/j.jim.2007.07.015.

Nielsen, M., Marcatili, P., 2015. Prediction of antibody epitopes. Methods Mol. Biol. 1348, 23-32.

Nielsen, M., Lundegaard, C., Worning, P., et al., 2004. Improved prediction of MHC class I and class II epitopes using a novel Gibbs sampling approach. Bioinformatics 20 (9), 1388-1397. http://dx.doi.org/10.1093/bioinformatics/bth100.

R Development Core Team, 2007. R: a language and environment for statistical computing. http://www.R-project.org.

Roederer, M., Koup, R.A., 2003. Optimized determination of T cell epitope responses. J. Immunol. Methods 274 (1-2), 221-228.

RStudio, Inc. shiny, 2014. Easy web applications in R URL: http://shiny.rstudio.com.

Zhao, S., Zhai, S., Zhuang, Y., et al., 2007. Inter-clade cross-reactivity of HIV-1-specific T cell responses in human immunodeficiency virus type 1 infection in China. Curr. HIV Res. 5 (2), 251-259. http://dx.doi.org/10.2174/157016207780076995. 